\title{
THE MONAD SYSTEM OF THE FINEST COMPATIBLE UNIFORM STRUCTURE
}

\section{WARD HENSON}

ABSTRACT. The methods of nonstandard analysis are used to study the finest uniform structure compatible with the topology on a given completely regular, Hausdorff space.

Let $(X, \tau)$ be a completely regular, Hausdorff space and let $\mathcal{F}$ be the finest uniform structure on $X$ which is compatible with $\pi$. If ${ }^{*} X$ is the set corresponding to $X$ in some enlargement, then $\mathcal{F}$ corresponds to a partition $\left\{\mu_{\mathcal{F}}(p): p \epsilon^{*} X\right\}$ of ${ }^{*} X$ in a familiar way [5], [6]. This partition may be described by

$$
\begin{aligned}
q \in \mu_{\mathcal{F}}(p) \Leftrightarrow & { }^{*} d(p, q) \text { is infinitesimal for every } \\
& \text { continuous pseudometric } d \text { on }(X, \tau) .
\end{aligned}
$$

The results in this paper concern the structure of the monad system $\mu_{\mathcal{F}}$, especially as it is related to the set $C(X)$ of continuous, real-valued functions on $(X, \tau)$. Also, it is proved that $\mu_{\mathcal{F}}$ is identical to the $\mu$-monad system constructed in a quite different way by Wattenberg [8] and some consequences of this fact are discussed.

In general we adopt in this paper the framework for nonstandard analysis which is described in Luxemburg's important paper [5]. Throughout this paper $M$ will denote a higher order set-theoretical structure and $* M$ will denote an enlargement of $\mathbb{M}$ which is $\boldsymbol{N}_{0}$-enlarging in the sense of [4]. Given a uniform space $(X, \mathcal{U})$ in $M$, we let $\stackrel{\underline{U}}{=}$ be the equivalence relation on ${ }^{*} X$ which corresponds to $\mathcal{U}$; that is

$$
p \stackrel{\text { u}}{=} q \Leftrightarrow(p, q) \in{ }^{*} V \text { for all } V \in U
$$

For each $p \in \epsilon^{*} X$ the $U$-monad of $p$, which is denoted by $\mu_{\mathcal{U}}(p)$, is the equivalence class of $p$ under $\stackrel{\underline{U}}{=}$. A discussion of how the monads $\mu_{\mathcal{U}}(p)$ form a basis for the nonstandard theory of uniform spaces may be found in [5] or [6]. 
A uniform structure $\tilde{\mathrm{U}}$ is generated on ${ }^{*} X$ by the collection $\left\{{ }^{*} V: V \epsilon\right.$ U\} of subsets of ${ }^{*} X \times{ }^{*} X$, as described in [3]. For each $p \in{ }^{*} X$, the monad

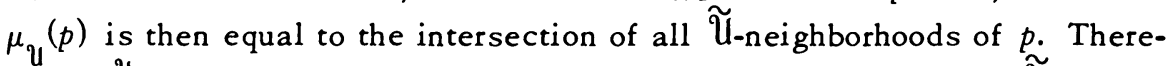
fore, $p \stackrel{\underline{U}}{=} q$ if and only if $p$ and $q$ have exactly the same filter of $\widetilde{U}_{\text {-neigh- }}$ borhoods. Let $X_{0}=\left\{\mu_{\mathrm{U}}(p): p \in{ }^{*} X\right\}$ and let $\mathcal{U}_{0}$ be the quotient uniformity induced on $x_{0}$ by $\widetilde{\mathrm{U}}$. Denote the quotient map from $\left({ }^{*} x, \widetilde{\mathrm{U}}\right)$ onto $\left(x_{0}, \mathcal{U}_{0}\right)$ by $\pi_{u}$.

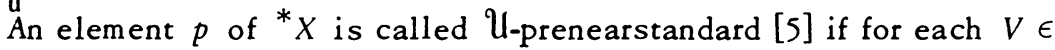
$\mathcal{U}$ there exists $x \in X$ with $\left(p,{ }^{*} x\right) \epsilon^{*} V$. The set of U-prenearstandard points is denoted by pns ${ }_{\mathcal{U}}$. Note that pns, $\mathrm{U}$ is just the $\widetilde{\mathrm{U}}$-closure in ${ }^{*} X$ of the set $\left\{{ }^{*} x: x \in X\right\}$ of standard points in ${ }^{*} X$. It can be shown that $\pi_{\mathrm{U}}$ (pns $\mathrm{u}_{\mathrm{U}}$ ) is $\mathcal{U}_{0}$-complete so that if $(X, \mathcal{U})$ is Hausdorff, then the subspace $\pi_{u}\left(\right.$ pns $\left._{\mathcal{U}}\right)$ of $\left(X_{0}, \mathcal{U}_{0}\right)$ is the completion of $(X, \mathcal{U})$.

Our purpose in assuming that ${ }^{*} M$ is $X_{0}$-enlarging is to obtain a local description of the $\widetilde{U}_{\text {-topology on }}{ }^{*} X$ in terms of the U-monads, as given in Theorem 1. Recall that $* M$ is $X_{0}$-enlarging if and only if it has the following property described in [5]: if $Y$ is a set in $\mathbb{M}, \varrho$ is a filter on $Y$ and $A$ is an internal subset of ${ }^{*} Y$, then $A \cap \mu(\zeta) \neq \varnothing$ if and only if $A \cap^{*} W \neq \varnothing$ for every $W \in \mathcal{G}$. (Here $\mu(\mathcal{G})$ is the filter monad of $\mathcal{G}$ defined by $\mu(\mathcal{G})=$ $\left.\bigcap{ }^{*} w: w \in \mathcal{G}\right\}$.) Recall also that for each set $X$ there exists $* M$ which is an $\boldsymbol{X}_{0}$-enlarging extension of some $\mathbb{M}$ containing $X$. (For example, the limit of a sequence of successive enlargements, the first of which has $X$ as an element.)

Theorem 1. Let $(X, \mathcal{U})$ be a uniform space in $M$.

(i) If $A \subseteq{ }^{*} X$ is internal, then the interior of $A$ in the $\tilde{U}$-topology is $\left\{p: \mu_{u}(p) \subseteq A\right\}$.

(ii) For $p \epsilon^{*} X$, a basis for the $\widetilde{\mathrm{U}}_{\text {-neighborhood filter at } p \text { consists of }}$ those internal subsets of ${ }^{*} X$ which contain $\mu_{\eta}(p)$.

Proof. (i) If $p$ is in the $\widetilde{U}_{\text {-interior of } A \text {, then }}{ }^{*} V(p) \subseteq A$ for some $V \in \mathcal{U}$ and therefore $\mu_{\mathrm{U}}(p) \subseteq A$. If $p$ is not in the interior of $A$, then ${ }^{*} V(p)$ intersects ${ }^{*} X \sim A$ for every $V \in \mathcal{U}$. Since ${ }^{*} \pi$ is $\boldsymbol{x}_{0}$-enlarging it follows that the filter monad of $\mathcal{U}$, which is equal to the equivalence relation $\stackrel{\underline{U}}{=}$, intersects $\left({ }^{*} X \sim A\right) \times\{p\}$. Therefore $\mu_{\mathcal{U}}(p) \not \subset A$ if $p$ is not in the interior of A.

(ii) This is an immediate consequence of (i) and the fact that the

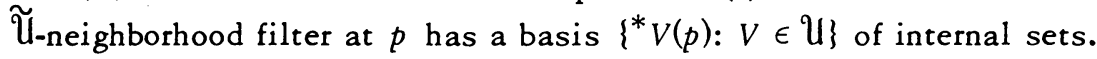


The observations in Theorem 1 are useful in the following type of setting: $\mathcal{U}$ and $\mathcal{C}$ be two uniform structures on $X$ and suppose that $S$ is a subset of ${ }^{*} X$ such that $\mu_{u}(p) \subseteq \mu_{0}(p)$ for every $p \in S$. Then there is a natural function $\phi$ from $\pi_{u}(S)$ onto $\pi_{\vartheta}(S)$ which takes $\pi_{u}(p)$ to $\pi_{\vartheta}(p)$ for each $p \in S$. Theorem 1 implies that the $\widetilde{U}_{\text {-topology is finer than the }}$ $\widetilde{C}$-topology when restricted to $S$. It follows that $\phi$ is continuous (relative to the $\mathcal{U}_{0}$-topology on $\pi_{\mathcal{U}}(S)$ and the $\mathcal{C}_{0}$-topology on $\left.\pi_{\tau}(S)\right)$. In particular, if $\mu_{u}(p)=\mu_{\vartheta}(p)$ for every $p \in S$, then $\phi$ is a homeomorphism.

Now let $(X, \tau)$ be a completely regular, Hausdorff space in $M$ and let $\mathcal{F}$ be the finest compatible uniform structure on $(X, \tau)$. In the next result, the difficult part of which is a consequence of Shirota's theorem [2, Theorem 15.21], the relation between $\mathcal{F}$ and $C(X)$ is explored in terms of nonstandard analysis.

Theorem 2. For each $p \in X^{*}$

$$
\mu_{\mathcal{F}}(p) \subseteq\left\{q:{ }^{*} f(p)={ }_{1}{ }^{*} f(q) \text { for all } f \in C(X)\right\} .
$$

If $p$ is $\mathcal{F}$-prenearstandard, then equality holds in (1). Moreover, if $(X, \tau)$ has no closed, discrete subspace of measurable cardinality, then

$$
\text { pns }_{\mathbf{Y}}=\left\{p:{ }^{*} f(p) \text { is finite for all } f \in C(X)\right\} \text {. }
$$

Proof: For each $f \in C(X)$ the function $|f(x)-f(y)|$ is a continuous pseudometric on $(X, \tau)$. Therefore $p \stackrel{\mathcal{F}}{=} q$ implies ${ }^{*} f(p)={ }_{1} * f(q)$ for any $p, q \in X$ and $f \in C(X)$. This shows that (1) holds in general.

To prove that equality holds in (1) for prenearstandard points, let $p \in$ pns $_{\mathcal{F}}$. If $q \notin \mu_{\mathcal{G}}(p)$, then for some standard $\delta>0$ and some continuous pseudometric $d$ on $(X, \tau),{ }^{*} d(p, q)>\delta$. Since $p \in$ pns $_{\mathfrak{f}}$ there exists $x \in X$ which satisfies ${ }^{*} d\left(p,{ }^{*} x\right)<\delta / 3$. The function $f(y)=d(y, x)$ is in $C(X)$, but ${ }^{*} f(p)$ cannot be infinitely close to ${ }^{*} f(q)$ (since otherwise ${ }^{*} d\left(q,{ }^{*} x\right)<$ $\delta / 2$ and hence $\left.{ }^{*} d(p, q)<\delta\right)$. Thus $q$ is also not in the set on the right side of (1).

Now let $\mathcal{U}$ be the uniform structure on $X$ generated by the pseudometrics $|f(x)-f(y)|$ for $f \in C(X)$. Evidently $U \subseteq \mathcal{F}$ and therefore pns $\subseteq_{\mathcal{F}} \subseteq$ pns $\mathcal{U}$. Moreover, pns ${ }_{q}$ is equal to the right side of (2) by [5, Theorem 3.15.5]. By the remarks above, a homeomorphism $\phi$ of $\pi_{c y}$ (pns $s_{c f}$ ) into $\pi_{u}$ (pns $\left.s_{u}\right)$ may be defined by setting $\phi\left(\pi_{\mathcal{F}}(p)\right)$ equal to $\pi_{\mathcal{U}}(p)$ for $p \in$ pns $s_{\mathcal{F}}$. Now $\pi_{\mathfrak{F}}\left(p^{\prime} s_{\mathcal{F}}\right)$ is the completion of $(X, \mathcal{F})$ and $\pi_{\mathcal{U}}\left(\mathrm{pns}_{\mathcal{u}}\right)$ is the completion of $(X, \mathcal{U})$. In case the stated assumption holds, then Shirota's theorem [2, 
Theorem 15.21] implies that both of these completions are real compact. Also they both contain $X$ densely and $\phi$ is the identity map on $X$. Since every function in $C(X)$ extends to a continuous function on $\pi_{\mathcal{u}}\left(\mathrm{pns}_{\mathrm{u}}\right)$ it follows that $\phi$ maps $\pi_{\mathcal{F f}}\left(\mathrm{pns}_{\mathcal{G F}}\right)$ onto $\pi_{\mathcal{U}}$ (pns $\mathrm{p}_{\mathrm{U}}$ ). The first part of the theorem then implies (2).

Next we show that the uniform structure $\mathcal{F}$ is closely related to the monad systems introduced by Wattenberg in [7] and [8]. The most interesting of these systems, which we will call the $\mu$-monad system, is constructed as follows [8, Definition 2.9]: given a completely regular, Hausdorff space $(X, \tau)$ in $M$, let $\lambda$ be a cardinal number which is greater than $2 X_{0}$ and greater than the cardinality of $X$. Let $S$ be a set of cardinality $\lambda$ and let $\mathbf{R}^{\lambda}$ be the set of real-valued functions $\alpha$ on $S$ whose support $\{s: \alpha(s)$ $\neq 0\}$ is finite. Equip $\mathbf{R}^{\lambda}$ with the metric $d_{\lambda}$ defined by

$$
d_{\lambda}(\alpha, \beta)=\sup \{|\alpha(s)-\beta(s)|: s \in S\} .
$$

The $\mu$-monad for $(X, \tau)$ consists of the family $\left\{\mu(p): p \epsilon^{*} X\right\}$ of subsets of ${ }^{*} X$ defined by

$$
\begin{aligned}
& \mu(p)=\{q: \text { for each continuous function } \\
& \left.\qquad f:(X, \tau) \rightarrow\left(\mathbf{R}^{\lambda}, d_{\lambda}\right),{ }^{*} d_{\lambda}\left({ }^{*} f p,{ }^{*} f q\right)={ }_{1} 0\right\} .
\end{aligned}
$$

The significance of the $\mu$-monad system lies in these facts, proved in [8]:

(i) $\left\{\mu(p): p \epsilon^{*} X\right\}$ is a partition of ${ }^{*} X$ which agrees with the $\tau$-monad partition on $r$-nearstandard points;

(ii) if $f:\left(X_{1}, \tau_{1}\right) \rightarrow\left(X_{2}, r_{2}\right)$ is a continuous function between completely regular, Hausdorff spaces in $\mathbb{M}$, then for each $p \in{ }^{*} X_{1},{ }^{*} f(\mu(p)) \subseteq \mu\left({ }^{*} f(p)\right)$. Moreover, the $\mu$-monad system is the same as the metric monad system on metric spaces and is the same as the covering monad system on normal spaces [8, Theorem 2.10], both of which have topologically natural definitions.

Our next result is that the $\mu$-monad on ${ }^{*} X$ is identical to the monad $\mu_{\mathcal{F}}$ given by the finest compatible uniform structure $\mathcal{F}$ on $(X, \tau)$. This theorem not only makes a connection between Wattenberg's work and the theory of uniform spaces, but is also leads (via his work) to useful descriptions of the $\mu_{\mathrm{cf}}$-monads in certain special situations.

Theorem 3. If $(X, \tau)$ is a completely regular, Hausdorff space in $\mathbb{M}$ and $\mathcal{F}$ is the finest compatible uniform structure on $(X, \tau)$, then $\mu(p)=$ $\mu_{f f}(p)$ for all $p \in{ }^{*} X$. 
Proof. Let the $\mu$-monad for $(X, \tau)$ be constructed as described above. The definition of $\mu(p)$ for $p \in{ }^{*} X$ makes it evident that there is a filter $\mathcal{U}$ on $X \times X$ such that the filter monad of $\mathcal{U}$ equals $\{(p, q): q \in \mu(p)\}$. Since this set is an equivalence relation on ${ }^{*} X$, it follows that $\mathcal{U}$ is a uniform structure on $X$ [5, Theorem 3.9.1]. In addition, $\mu(p)=\mu_{u}(p)$ for all $p \epsilon^{*} X$. By (i) above, for each $x \in X$

$$
\mu_{\mathcal{U}}(x)=\{p: p \text { is } \tau \text {-nearstandard to } x\}=\mu_{\tau}(x) .
$$

This implies that the uniform structure $\mathcal{U}$ is compatible with $\tau$ and hence $\mathcal{U} \subseteq \mathcal{F}$.

Now $\mathcal{F}$ may be described as the coarsest uniform structure on $X$ for which every continuous function from $(X, r)$ into a metric space is actually uniformly continuous. Let $(Y, d)$ be a metric space and $f:(X, \tau) \rightarrow(Y, d)$ a continuous function. To prove that $f$ is uniformly continuous relative to $\mathcal{U}$ it suffices to show

$$
{ }^{*} f(\mu(p)) \subseteq\left\{q:{ }^{*} d\left(q,{ }^{*} f p\right)={ }_{1} 0\right\}
$$

for each $p \epsilon^{*} X$. Property (ii) of the $\mu$-monad system implies that ${ }^{*} f(\mu(p))$ $\subseteq \mu\left(^{*} f(p)\right)$. But the $\mu$-monad for $(Y, d)$ is the same as the metric monad [8, Theorem 2.10] which is finer than the $d$-monad. That is

$$
\mu\left({ }^{*} f(p)\right) \subseteq\left\{q:^{*} d\left(q,{ }^{*} f p\right)={ }_{1} 0\right\}
$$

which yields (3). That is, each continuous function from $(X, \tau)$ into a metric space is uniformly continuous relative to $\mathcal{U}$, and therefore $\mathcal{F} \subseteq \mathcal{U}$. This proves $\mathcal{U}=\mathcal{F}$ so that $\mu(p)=\mu_{\mathcal{U}}(p)=\mu_{\mathcal{G F}}(p)$ for every $p \epsilon^{*} X$.

Corollary 1. If $(X, d)$ is a metric space in $\mathbb{M}$ and $\mathcal{F}$ is the finest uniform structure compatible with the d-topology on $X$, then for $p \epsilon^{*} X$

$$
\mu_{\mathcal{F}}(p)=\left\{q:{ }^{*} d(p, q)<{ }^{*} f(p) \text { for every positive function } f \text { in } C(X)\right\} \text {. }
$$

Proof. Theorem 3 and [8, Theorem 2.10].

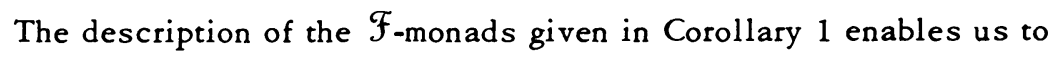
give a simple proof of a result for subspaces of the Euclidean spaces which is contained in the paper [1] of Corson and Isbell.

Corollary 2. If $(X, \tau)$ is homeomorphic to a subspace of $\mathbf{R}^{n}$ for some $n \geq 1$ and $\mathcal{F}$ is the finest compatible uniform structure on $(X, \tau)$, then $\mathcal{F}$ is the uniform structure on $X$ generated by the pseudometrics $|f(x)-f(y)|$ for $f \in C(X)$. That is, $\mathcal{F}$ is the unique compatible uniform structure on 
$(X, \tau)$ which makes every function in $C(X)$ uniformly continuous.

Proof. We assume $X \subseteq \mathbf{R}^{n}$ and let $\tau$ be the subspace topology so $\tau$ is defined by the metric

$$
d(x, y)=\max \left\{\left|x_{i}-y_{i}\right|: 1 \leq i \leq n\right\}
$$

where $x=\left(x_{1}, \ldots, x_{n}\right)$ and $y=\left(y_{1}, \ldots, y_{n}\right)$. By Corollary 1 it suffices to show that if ${ }^{*} f(p)={ }_{1}{ }^{*} f(q)$ for every $f \in C(X)$, then ${ }^{*} d(p, q)<{ }^{*} g(p)$ for every positive $g \in C(X)$. To prove this, let $p, q$ satisfy the first condition and let $g \in C(X)$ be positive. We will show ${ }^{*} d(p, q)<{ }^{*} g(p)$, and it may be assumed that $g$ is bounded on $X$. Then

$$
1={ }_{1} * g(q) /{ }^{*} g(p)
$$

since $1 / g(x)$ is in $C(X)$ and ${ }^{*} g(q)$ is finite. Let $p=\left(p_{1}, \ldots, p_{n}\right)$ and $q=\left(q_{1}, \ldots, q_{n}\right)$. Since $x_{i} / g(x)$ is in $C(X)$, it follows from (4) that

$$
p_{i} /{ }^{*} g(p)={ }_{1} q_{i} /^{*} g(p)
$$

This shows that $\left|p_{i}-q_{i}\right|<{ }^{*} g(p)$ for $1 \leq i \leq n$ and therefore ${ }^{*} d(p, q)<$ * $g(p)$, completing the proof.

Now let $(X, \tau)$ be an arbitrary completely regular, Hausdorff space in $M$ and let $\mathcal{F}$ be the finest compatible uniform structure on $(X, \tau)$. Two elements $p, q$ of ${ }^{*} X$ are in the same $\mathcal{F}$-galaxy [3] if for each $V \in \mathcal{F}$ there is a finite sequence $p_{0}, \ldots, p_{k}$ in ${ }^{*} X$ such that $p_{0}=p, p_{k}=q$ and $\left(p_{i}, p_{i+1}\right) \in{ }^{*} V$ for each $i=0, \ldots, k-1$. In particular this implies that ${ }^{*} d(p, q)$ is finite for every continuous pseudometric $d$ on $(X, \tau)$. Therefore, the set $A=\left\{p:{ }^{*} f(p)\right.$ is finite for all $\left.f \in C(X)\right\}$ is a union of $\mathcal{F}$-galaxies, as is ${ }^{*} X \sim A$.

If there are no measurable cardinals, then Theorem 2 shows that $\pi_{\mathrm{gf}}(A)$ is the completion of $(X, \mathfrak{F})$. Therefore the galaxy structure for points in $A$ reflects in an immediate way the structure of $(X, \tau)$. The next result shows that the galaxy structure for points outside $A$ is trivial. This has the immediate consequence that any connected subset of $X_{0}=\pi_{\mathfrak{F}}\left({ }^{*} X\right)$ with at least two elements must be contained in $\pi_{\mathcal{F}}(A)$. That is, $\pi_{\mathcal{F}}(A)$ is open and closed in $X_{0}$ and $X_{0} \sim \pi_{\mathcal{F f}}(A)$ is totally disconnected (in the $\mathcal{F}_{0}$-topology on $\left.X_{0}\right)$.

Theorem 4. Let $(X, \tau)$ be a completely regular, Hausdorff space in $\mathbb{M}$ and let $\mathcal{F}$ be the finest compatible uniform structure on $(X, \tau)$. If $p \in \epsilon^{*} X$ and there exists $f \in C(X)$ such that ${ }^{*} f(p)$ is infinite, then the $\mathcal{F}$-galaxy containing $p$ is equal to the monad $\mu_{\mathcal{G}}(p)$. 
Proof. Suppose $p \epsilon^{*} X$ and $f \in C(X)$ and assume that ${ }^{*} f(p)$ is infinite.

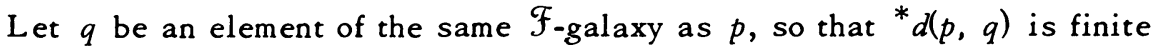
for every continuous pseudometric $d$ on $(X, \tau)$ [3, Theorem 4.2].

We may assume $f \geq 1$; let $g=1 / f \in C(X)$ so $0<g \leq 1$ and ${ }^{*} g(p)={ }_{1} 0$. Given a continuous pseudometric $d$, define

$$
d^{\prime}(x, y)=d(x, y)+|g(x)-g(y)|
$$

and

$$
V_{n}=\left\{(x, y): d^{\prime}(x, y)<n^{-1} \cdot g(x)\right\}
$$

for $n \geq 1$. Since $d^{\prime}$ is a continuous pseudometric on $(X, \tau)$, the sets $V_{n}$ are open neighborhoods of the diagonal in $X \times X$. We will show that $V_{n+1}$ $\subseteq\left(V_{n}\right)^{-1}$ and $\left(V_{4 n+1}\right)^{2} \subseteq V_{n}$ for each $n \geq 1$. Suppose $d^{\prime}(x, y)<(n+1)^{-1}$. $g(x)$. Then $|g(x)-g(y)|<(n+1)^{-1} \cdot g(x)$ so that $(n+1)^{-1} \cdot g(x)<n^{-1} g(y)$ and $g(y)<(n+2)(n+1)^{-1} \cdot g(x)$. It follows that $d^{\prime}(x, y)<n^{-1} g(y)$, which proves $V_{n+1} \subseteq\left(V_{n}\right)^{-1}$. If also $d^{\prime}(y, z)<(n+1)^{-1} \cdot g(y)$, then

$$
d^{\prime}(x, z)<\left((n+1)^{-1}+n^{-1}\right) g(y)<4 n^{-1} g(x) .
$$

This proves $\left(V_{4 n+1}\right)^{2} \subseteq V_{n}$. It follows from these facts that $\left\{V_{n}: n \geq 1\right\}$ generates a uniform structure on $X$ which defines a topology coarser than 7. Therefore $\left\{V_{n}: n \geq 1\right\}$ is contained in $\mathcal{F}$.

Now since $V_{1}$ is in $\mathcal{F}$ and since $p$ and $q$ are in the same $\mathcal{F}$-galaxy, there is a finite sequence $p_{0}, \ldots, p_{k}$ in ${ }^{*} X$ such that $p_{0}=p, p_{k}=q$ and for each $i=0, \ldots, k-1$

$$
{ }^{*} d\left(p_{i}, p_{i+1}\right)+\left|{ }^{*} g\left(p_{i}\right)-{ }^{*} g\left(p_{i+1}\right)\right|<{ }^{*} g\left(p_{i}\right) .
$$

An inductive argument shows that ${ }^{*} g\left(p_{i}\right)={ }_{1} 0$ for each $i=0, \ldots, k-1$. Using the triangle inequality for ${ }^{*} d$ yields

$$
{ }^{*} d(p, q)<\sum_{i=0}^{k-1} * g\left(p_{i}\right)={ }_{1} 0 .
$$

That is, ${ }^{*} d(p, q)={ }_{1} 0$ for every continuous pseudometric $d$ on $(X, \tau)$. Therefore $q \in \mu_{\mathcal{F}}(p)$ and the proof is complete.

\section{REFERENCES}

1. H. H. Corson and J. R. Isbell, Euclidean covers of topological spaces, Quart. J. Math. Oxford Ser. (2) 11 (1960), 34-42. MR 23 \#2194.

2. Leonard Gillman and Meyer Jerison, Rings of continuous functions, University Ser. in Higher Math., Van Nostrand, Princeton, N. J., 1960. MR 22 \#6994. 
3. C. Ward Henson, The nonstandard hulls of a uniform space, Pacific J. Math. 43 (1972), 115-137. MR 47 \# 2559.

4. C. Ward Henson and L. C. Moore, Jr., The nonstandard theory of topological vector spaces, Trans. Amer. Math. Soc. 172 (1972), 405-435. MR 46 \# 7836.

5. W. A. J. Luxemburg, A general theory of monads, Applications of Model Theory to Algebra, Analysis and Probability, Holt, Rinehart and Winston, New York, 1969, pp. 18-86. MR 39.\#6244.

6. M. Machover and J. Hirschfeld, Lectures on nonstandard analysis, Lecture Notes in Math., Vol. 94, Springer-Verlag, Berlin, 1969. MR 40 \# 2531.

7. Frank Wattenberg, Nonstandard topology and extensions of monad systems to infinite points, J. Symbolic Logic 36 (1971), 463-476. MR 45 \#2642.

8. - Monads of infinite points and finite product spaces, Trans. Amer. Math. Soc. 176 (1973), 351-368. MR 47 \# 1020.

DEPARTMENT OF MATHEMATICS, DUKE UNIVERSITY, DURHAM, NORTH CAROLINA 27706

Current address: Department of Mathematical Sciences, New Mexico State University, Las Cruces, New Mexico 88003 